\title{
Beyond-Line-of-Sight Ducting Channels: Coherence Bandwidth, Coherence Time and Rain Attenuation
}

\author{
Ergin Dinc, Student Member, IEEE, Ozgur B. Akan, Senior Member, IEEE
}

\begin{abstract}
Atmospheric ducts trap the propagating signals by refraction. In this way, the propagating signals in the duct can propagate through beyond-Line-of-Sight distances (b-LoS). Although atmospheric ducts are promising candidate for bLoS communication especially for military applications, channel characteristics of the ducting channel have not been completely modeled. In this paper, coherence time and coherence bandwidth of ducting channels are analyzed for the first time in the literature. In addition, a slant-path rain attenuation model is proposed to determine rain attenuation in ducting links for the first time. At the end, simulation results are presented for the distribution of rain loss and spectral efficiency of the system.
\end{abstract}

Index Terms-Communication channels, Propagation, Rain

\section{INTRODUCTION}

A TMOSPHERIC ducts are the layer in which propagating rays are trapped between surface and ducting layer due to the refractive effects of the lower troposphere [1]-[3]. As reviewed in [1], ducting channel can provide high data rate b-LoS communications especially in naval and maritime environments because duct formation is highly probable in the environments with high humidity.

In ducting communications, both of the antennas should be in the duct layer in order to utilize the trapping effect. In this way, the trapped signals can propagate through b-LoS ranges because ducting layer avoids the spreading of signal energy to the atmosphere. Instead, signal energy is concentrated between surface and duct layer. Ducting channel communication can be utilized as a communication medium up to $400 \mathrm{~km}$ [1], [4]. Trapping effect of atmospheric ducts are effective between $5-10.5 \mathrm{GHz}$ depending on duct properties. Since atmospheric ducts utilize such high frequency range and signal rays follow high distances close to the surface level, ducting channel may be exposed to rain attenuation. However, there is no study which considers rain attenuation in b-LoS ducting links.

There are plenty of rain attenuation models in the literature [5]-[7]. Most of the available studies consider the slant-path rain attenuation models for satellite communications (SATCOM) [6], [7]. The closest work for this paper is [8] in which a slant-path rain attenuation model is proposed for troposcatter considering nonuniform rainfall distribution. We utilize similar rain data generation as in [8], but the path characteristics are completely different in ducting channels. Thus, the model in [8] is not applicable to ducting channels and there is no study which considers rain attenuation in ducting links.

E. Dinc and O. B. Akan are with the Next-generation and Wireless Communications Laboratory, Department of Electrical and Electronics Engineering, Koc University, Istanbul, 34450 Turkey (e-mail: edinc@ku.edu.tr; akan@ku.edu.tr).
The main contributions of this paper are two-fold. Firstly, coherence bandwidth and time of ducting channels are analyzed. In [4], the authors analyze delay spread of the channel with frequency independent ray-optics methods. In this paper, coherence bandwidth is determined via frequency dependent frequency correlation results. In addition, coherence time of the channel is estimated by utilizing angle constrains developed in [4]. Coherence bandwidth and time analysis can be utilized to determine the bandwidth and mobility levels in which the b-LoS channel has flat and slow fading. Secondly, a slant-path rain attenuation model for ducting channel is developed based on the ray analysis in [4] and synthetic rain distribution model in [9], [10]. Simulation results are presented for the rain loss and spectral efficiency of the system under rain. These results can guide the b-LoS system designer to determine the carrier frequency which provides better spectral efficiencies with or without rain to design more reliable links.

\section{ATMOSPheric Ducts}

Atmospheric duct is the layer in which modified refractivity gradient is turned negative to positive $(0 \geq \partial M / \partial z)$ as in Figure 1(a). This layer traps the propagating rays with low grazing angles, and the trapped signal energy is concentrated between the surface and duct layer. In this way, propagating signals can reach b-LoS distances [1], [3]. Since duct formation is more probable in humid areas, ducting channel is a promising b-LoS communication medium especially in naval communication applications [1]. In maritime and coastal areas, the occurrence probability of atmospheric ducts varies between $60-80 \%$ according to the season [11].

Atmospheric ducts can be divided into four categories according to their formation process: evaporation ducts, surfacebased ducts, elevated ducts, and surface ducts. In [1], the authors analyze path-loss of a $13 \mathrm{~m}$ evaporation duct and show that $10.5 \mathrm{GHz}$ frequency has the lowest path-loss. In [4], the authors develop a large-scale path-loss model for a $40 \mathrm{~m}$ surface duct. According to their results, lower frequency ranges $(3-5 \mathrm{GHz})$ has better trapping effects. The difference between frequency range is caused by duct height difference. As experimentally presented in [12], evaporation ducts with high duct heights also perform better around $3 \mathrm{GHz}$. Since modified refractivity profiles of evaporation duct and surface duct are similar as shown in Figure 1(a), we can approximate evaporation ducts with a surface duct with the same duct height and duct strength. Duct strength is the change in modified refractivity from surface to trapping layer. For this reason, the analysis presented within this paper is also valid for the evaporation duct with the same duct parameters. 


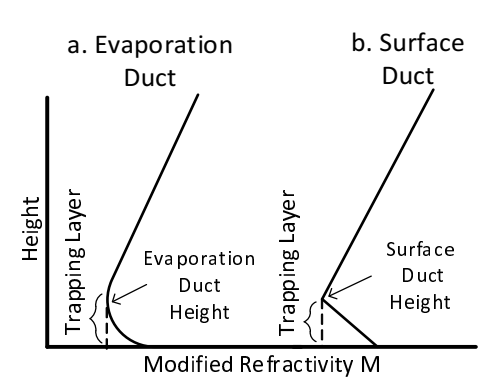

(a)

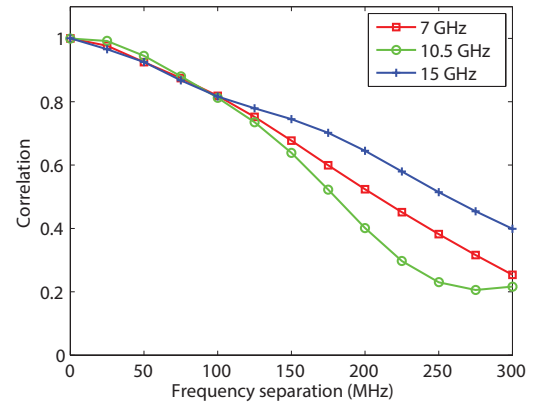

(b)

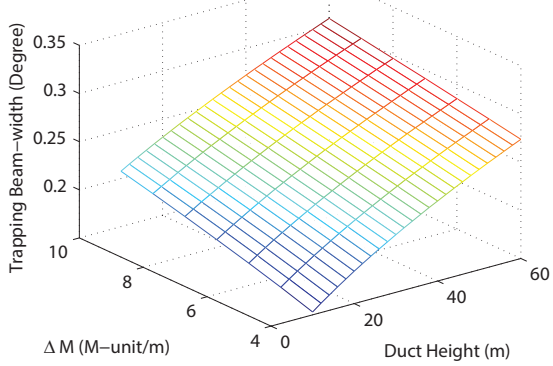

(c)

Fig. 1. (a) Modified refractivity profile of evaporation duct and surface duct, (b) coherence bandwidth for ducting links, (c) trapping beam-width values for $5 \mathrm{~m}$ antenna height.

\section{COHERENCE BANDWIDTH AND TIME}

In this section, the analysis for coherence bandwidth and coherence time of ducting links are presented.

\section{A. Coherence Bandwidth}

In [4], maximum delay spread of the channel is estimated via ray-optics methods which are independent of frequency. To this end, the coherence bandwidth of the ducting channel is analyzed with parabolic equation (PE) methods to observe the effects of frequency. For this purpose, we use PETOOL [13] which is a split-step-Fourier based PE wave propagation tool to estimate the path-loss in ducting links. PETOOL is calibrated with experimentally validated tools [13].

PETOOL results are utilized to estimate the coherence bandwidth in ducting channels. To this end, we generate two path-loss profiles that have some frequency separation and calculate the correlation between vertical cuts at the desired range to find the frequency correlation. Figure 1(b) shows the correlation vs. frequency separation for the surface duct with the following simulation parameters: $150 \mathrm{~km}$ range, $20 \mathrm{~m}$ transmitter and receiver height, duct height $40 \mathrm{~m}$, duct strength 10 M-units and horizontal polarization. The channel can be assumed as flat when the frequency correlation is lower than 0.5. As in Figure 1(b), the ducting channel can be assumed as a flat-fading channel if the channel bandwidth is lower than $205 \mathrm{MHz}$ for $7 \mathrm{GHz}, 180 \mathrm{MHz}$ for $10.5 \mathrm{GHz}$ and $252 \mathrm{MHz}$ for $15 \mathrm{GHz}$. According to the ray optics analysis presented in [4], ducting channels have coherence bandwidth levels more than $200 \mathrm{MHz}$. Therefore, our results are consistent with [4]. In addition, the frequency correlation do not show a notable pattern with the frequency because trapping effect also do not show direct relationship with frequency as in [1].

\section{B. Coherence Time}

For coherence time estimation, a simple Doppler spread approach is used based on the limited angle-of-arrival (AoA) range. Since ducting channel traps the signal at the ducting layer, only the rays that have particular angle range can be received at the receiver. In [4], an analytical formula for the trapping angle range is derived. Upper and lower bound for the trapping angle in the elevation cut can be estimated with

$$
\theta_{\text {max,min }}^{T}= \pm \sqrt{2\left(\frac{1}{n(0)} \frac{d n}{d z}-\frac{1}{R_{0}}\right)\left(h_{t}-\delta\right)},
$$

where $d n / d z$ is the refractive index derivative with respect to height, $h_{t}$ is the transmitter height, $\delta$ is the ducts height, $R_{0}$ is the earth radius $(\approx 6370 \mathrm{~km})$ and $n(0)$ is the index of refraction at surface $(\approx 1.00035)$ [1]. Atmospheric ducts are generally denoted with duct strength $(\Delta M)$ which is the change in the modified refractivity from bottom to top of the duct $\left(d n / d z=-10^{-6}(\Delta M / \delta-0.157)\right)$ [1].

Figure 1(c) shows the trapping beam-width values $\left(\theta_{\max }\right)$ for $5 \mathrm{~m}$ transmitter height. Based on experimental observations [11], [14], duct heights and strengths mostly vary between $10-40 \mathrm{~m}$ and $4-10 \mathrm{M}$-units, respectively. As in Figure $1(\mathrm{c})$, these duct height and strength ranges are associated with $0.2^{\circ}-0.3^{\circ}$ beam-width in the elevation cut. Thus, ducting channel has very limited receive angle span.

In ducting channel, the elevation cut has very narrow beamwidths $\left(0.2^{\circ}-0.3^{\circ}\right)$. Therefore, Doppler spread due to mobility is very limited in ducting channels and it is given as

$$
D_{s}=v \cos (\theta) / \lambda,
$$

where $v$ is the speed, $\lambda$ is the wavelength and $\theta$ is the angle between the received ray and horizon. We assume that the receiver is moving away from the transmitter on the same line. For $10.5 \mathrm{GHz}$ and $v=10 \mathrm{~m} / \mathrm{s}$, the Doppler spread becomes $349.9979 \mathrm{~Hz}$ for the $0.2^{\circ}$ ray and $349.9952 \mathrm{~Hz}$ for the $0.3^{\circ}$ ray. Therefore, the Doppler spread can be assumed as $\approx 350 \mathrm{~Hz}$ for $v=10 \mathrm{~m} / \mathrm{s}$ and the estimated coherence time is $\approx 2.9 \mathrm{~ms}$. Since the speed of naval vehicles will be not so high, coherence time of ducting links will be in the order of milliseconds. Thus, ducting channel will experience slow fading for $\mathrm{MHz}$ level bandwidths.

\section{Rain Attenuation Model}

Ducting links will utilize high frequency ranges as analyzed in [1]. Thus, b-LoS paths may experience strong rain attenuation. Path-loss and rain loss in the ducting links are mutually exclusive as outlined in [15]. Therefore, the total transmission loss can be determined by calculating path-loss and rain loss 


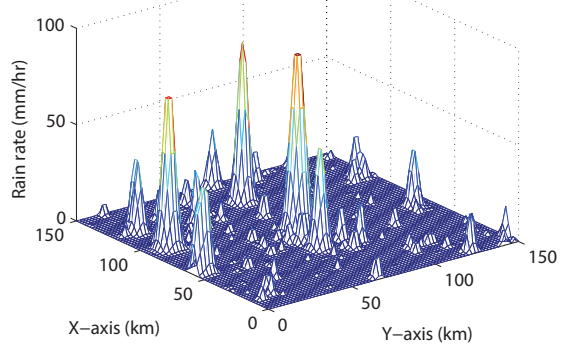

(a)

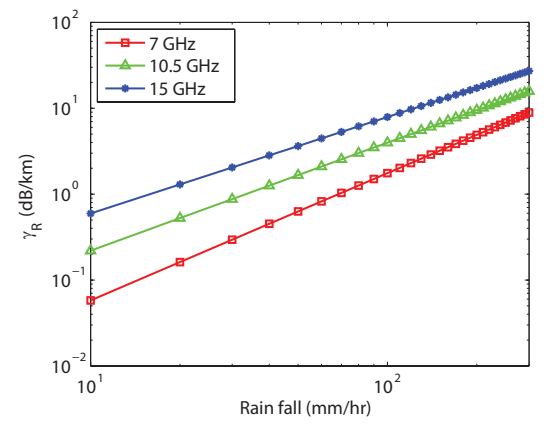

(b)

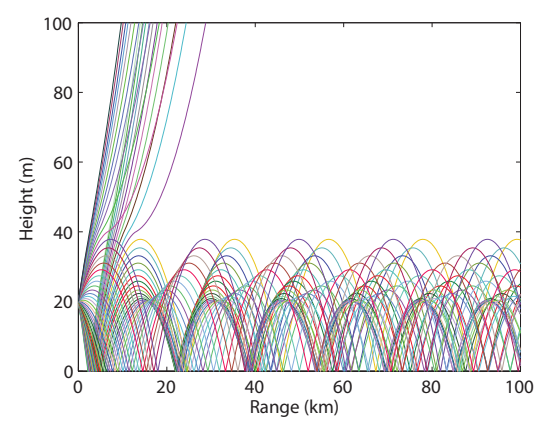

(c)

Fig. 2. (a) Spatial distribution of rain cells, (b) rain specific attenuation, (c) ducting channel ray trajectories.

separately. To this end, a rain attenuation model for ducting links is developed in this section.

\section{A. Rain Model}

A synthetic rain model is utilize to generate rain cell spatial distribution as in [8]. To this end, HYCELL model [9], [10] is adapted with a slight modification. In the HYCELL model, rain distribution in a single rain cell is modeled with both Gaussian and exponential functions. Since the exponential function models only tail of the rain cell, rainfall rates associated with exponential function is very low. Thus, only Gaussian function is utilized to model the rain cells in this paper, and rainfall distribution of a single cell is given as

$$
R(x, y)= \begin{cases}R_{G} \exp \left[-\left(x^{2} / a_{G}^{2}+y^{2} / b_{G}^{2}\right)\right], & \text { if } R>R_{1} \\ 0, & \text { otherwise }\end{cases}
$$

where $R_{G}$ is the peak rainfall rate, $a_{G}$ and $b_{G}$ are the distance where the rainfall rate decreases by a factor of $1 / e$ in $\mathrm{x}$ and $\mathrm{y}$ axis. Rain cells are assumed as symmetrical, such that $a_{G}=$ $b_{G}$. The minimum rain rate $\left(R_{1}\right)$ is utilized as $1 \mathrm{~mm} / \mathrm{hr}$ [9]. HYCELL model [9], [10] estimates spatial rain distribution based on local spatial rain probabilities which are provided by ITU-R P. 837-6 [16]. By utilizing the modified HYCELL model and local rain probabilities for Istanbul, Turkey [16], the spatial rain distribution is estimated as in Figure 2(a).

\section{B. Slant-path Model}

In this subsection, a slant-path rain attenuation model is developed for ducting channels by utilizing the rain model discussed in the previous part. In the slant-path model, ITU-R P.838 [17] rain specific attenuation is utilized and it represents the amount of path-loss in $\mathrm{dB} / \mathrm{km}$ due to rain as

$$
\gamma_{R}=k R^{\gamma} \mathrm{dB} / \mathrm{km},
$$

where $k$ and $\gamma$ are empirical coefficients and their values are utilized as provided in [17]. Figure 2(b) presents rain specific attenuation values for different frequencies. In the slant-path model, total rain attenuation is calculated by multiplying the rain specific attenuation with the rain path length. Thus, rain path length in ducting links is required to find the rain loss.

For rain path lengths, the ray trajectory analysis are utilized as introduced in [4]. Figure 2(c) shows the ray trajectories for the ducting channel with $20 \mathrm{~m}$ transmitter height, 40 $\mathrm{m}$ duct height and $10 \mathrm{M}$-units duct strength. The trapped rays follow longer paths compared to the range which is assumed as the horizontal distance between transmitter and receiver. However, the difference between ray path lengths and range is very low as presented in Figure 2(c). In the simulations, the average ratio of ray path length to the range is $\epsilon=1.00000635548381$. Thus, the parabolic shape of the rays can be neglected and the rain attenuation calculations may utilize direct rays between transmitter and receiver. To this end, we multiply the calculated path lengths with $\epsilon$.

In the proposed rain attenuation model, the rain attenuation for $i$ th rain cell can be calculated as

$$
\begin{aligned}
A_{\text {rain }}^{(i)} & =\int_{d_{s}}^{d_{e}} k R(x)^{\gamma} \epsilon d x \\
& =\int_{d_{s}}^{d_{e}} R_{G}^{\gamma} \exp \left(-\left(x-d_{m}\right)^{2} / a_{G}^{2}\right)^{\gamma} \epsilon d x \mathrm{~dB}, \text { (5) }
\end{aligned}
$$

where $d_{s}$ is the staring point of the rain cell, $d_{e}$ is the end point of the rain cell and $d_{m}=\left(d_{s}+d_{e}\right) / 2$ is the middle of the rain cell. By considering all of the rain cells in the communication path, the total amount of rain attenuation can be found as

$$
A_{\text {rain }}=\sum_{i=1}^{N_{R}} A_{\text {rain }}^{(i)} \mathrm{dB},
$$

where $N_{R}$ is the number of rain cells on the path.

\section{Spectral EFFiciency}

The spectral efficiency results are also provided for ducting links in the presence of rain in Section VI. To this end, this section includes the spectral efficiency calculations by utilizing the path-loss model developed in [4]. In single-input singleoutput links, the spectral efficiency can be found as

$$
R(t)=\log _{2}\left(1+\rho \times P_{t} / N\right) \text { bps, }
$$

where $P_{t}$ is the transmit power, $\rho$ is the path-loss which is calculated with the path-loss model in [4] and rain attenuation is also added to this term. $N$ is the noise power which is modeled as thermal noise $N=k B T$ where $T$ is the temperature in $\mathrm{K}, B$ is the bandwidth and $k$ is the Boltzmann constant. 


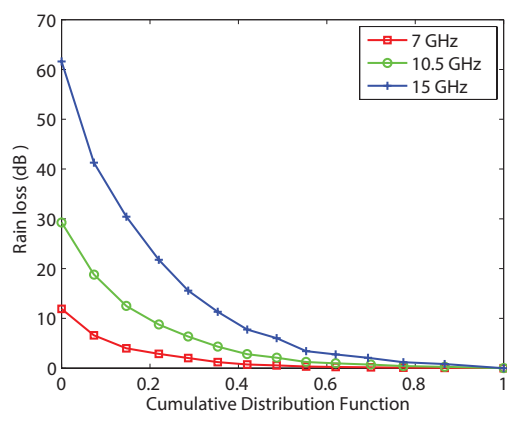

(a)

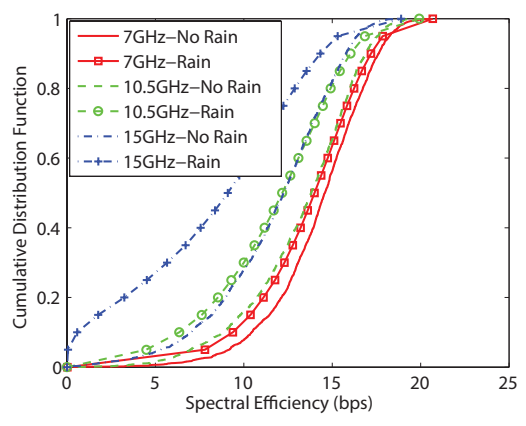

(b)

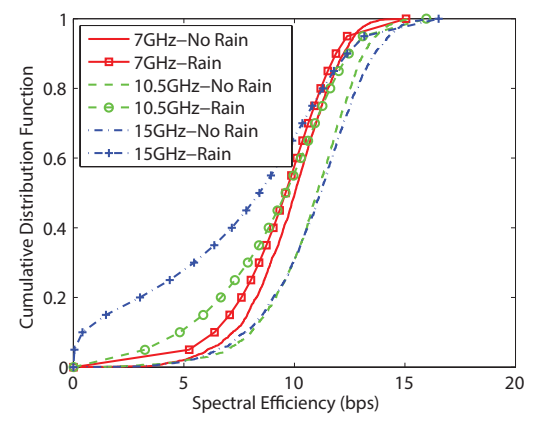

(c)

Fig. 3. (a) Rain loss in ducting links, (b,c) Spectral efficiency in ducting links for scenario 1 and 2.

\section{Simulation Results}

Rain attenuation for each ray is calculated with the slantpath model for the rainfall distribution in Figure 2(a). The rain loss is calculated for each $500 \mathrm{~m}$ cut in y-axis of the synthetic rainfall distribution in Figure 2(a). In this way, cumulative distribution function (CDF) of rain loss is calculated with the following link parameters: $40 \mathrm{~m}$ duct height, $10 \mathrm{M}$-units duct height, $150 \mathrm{~km}$ range, $20 \mathrm{~m}$ antenna heights, $1.2 \mathrm{~m}$ antenna diameter $\left(D_{a n t}\right)$ and 0.65 antenna efficiency $\left(\eta_{\text {ant }}\right)$.

TABLE I

Simulation PARAMETERS.

\begin{tabular}{|c|c|c|c|c|}
\cline { 3 - 5 } \multicolumn{1}{c|}{} & \multicolumn{2}{c|}{ Scenario 1 } & \multicolumn{2}{c|}{ Scenario 2 } \\
\hline Frequecy & T. Power & Bandwidth & T. Power & Bandwidth \\
\hline $7 \mathrm{GHz}$ & \multirow{3}{*}{$30 \mathrm{dBm}$} & \multirow{3}{*}{$20 \mathrm{MHz}$} & $45 \mathrm{dBm}$ & $13 \mathrm{MHz}$ \\
\cline { 1 - 3 } \cline { 4 - 5 } & & $30 \mathrm{dBm}$ & $20 \mathrm{MHz}$ \\
\cline { 4 - 5 } & & & $21 \mathrm{dBm}$ & $28 \mathrm{MHz}$ \\
\hline
\end{tabular}

Figure 3(a) shows the distribution of rain loss in a $150 \mathrm{~km}$ ducting link by utilizing the proposed slant-path rain model. As noticed, ducting channel can experience very high rain attenuations especially for frequencies higher than $10 \mathrm{GHz}$. By considering the high path-loss enhancements near $10.5 \mathrm{GHz}$ [1], ducting channel links is prone to rain loss.

In addition, we present the spectral efficiency distributions for two scenarios in Figure 3(b) and 3(c). In the first scenario, the same channel parameters is utilized for each frequency as in Table I with $40 \mathrm{~dB}$ antenna gains. As noticed in Figure 3 (b), rain attenuation may cause significant reduction in the spectral efficiency in high frequencies. In the second scenario, channel parameters are also changed in accordance with the carrier frequency as in Table I. In addition, the antenna gains are calculated with the generic equation for the parabolic reflector antennas $G=10 \log \left[\eta_{\text {ant }}\left(\pi D_{\text {ant }} \lambda^{2}\right)\right]$. Since higher antenna gains can be provided with higher carrier frequencies, the spectral efficiency performance of $10.5 \mathrm{GHz}$ and $15 \mathrm{GHz}$ becomes better without rain as presented in Figure 3(c). By considering the significant spectral efficiency loss in $15 \mathrm{GHz}$ due to rain, $10.5 \mathrm{GHz}$ is expected to provide higher data rates.

\section{CONCLUSION}

In this paper, the coherence bandwidth and time of the ducting links are analyzed. Our analyses show that ducting channel is expected to experience slow and narrow-band fading up to a few hundreds of $\mathrm{MHz}$ bandwidths. In addition, a slantpath rain attenuation model is proposed and, the amount of rain loss in the ducting links is presented. According to our results, $10.5 \mathrm{GHz}$ is more promising to provide higher data rates.

\section{REFERENCES}

[1] E. Dinc, O. B. Akan, "Beyond-Line-of-Sight Communications with Ducting Layer," IEEE Commun. Mag., vol. 52, pp. 37-43, Oct. 2014.

[2] M. J. Luddy, J. H. Winters, A. Lackpour. (2011). Beyond Line-ofSight Communications with Smart Antennas (BLoSSA) [online]. Available:http://www.atl.lmco.com/papers/1978.pdf.

[3] H. V. Hitney, J. H. Richter, R. A. Pappert, K. D. Anderson, G. B. Jr. Baumgartner, "Tropospheric radio propagation assessment," Proceedings of the IEEE, vol. 73, no. 2, Feb. 1985, pp. 265-283.

[4] E. Dinc, O. B. Akan, "Channel Model for the Surface Ducts: Large-scale Path-Loss, Delay Spread, and AOA," IEEE Transactions on Antennas and Propagation, vol. 63, no. 6, pp. 2728-2738, June 2015.

[5] "ITU-R's Rec. P. 839-3: Rain height model for prediction methods," 2001.

[6] Crane, R. K., Electromagnetic Wave Propagation Through Rain, Wiley Ser. Remote Sens., John Wiley, New York, 1996.

[7] A. Dissanayake, J, Allnutt, F. Haidara, "A prediction model that combines rain attenuation and other propagation impairments along Earth-satellite paths," IEEE Trans. Antennas Propag., vol. 45, no. 10, Oct. 1997.

[8] E. Dinc, O. B. Akan, "A Nonuniform Spatial Rain Attenuation Model for the Troposcatter Communication Links," to appear in IEEE Wireless Communications Letters, 2015.

[9] L. Feral, H. Sauvageot, L. Castanet, J. Lemorton, "HYCELL - A new hybrid model of the rain horizontal distribution for propagation studies: 1. Modeling of the rain cell," Radio Science, vol. 38, no. 3, 2003.

[10] L. Feral, H. Sauvageot, L. Castanet, J. Lemorton, "HYCELL - A new hybrid model of the rain horizontal distribution for propagation studies: 2. Statistical modeling of the rain rate field," Radio Sci., vol. 38, 2003.

[11] S. M. Babin "Surface Duct Height Distributions for Wallops Island, Virginia, 1985-1994,” J. Appl. Meteor., vol. 35, pp. 86-93, 1996.

[12] H. V. Hitney, R. Vieth, "Statistical assessment of evaporation duct propagation," IEEE Trans. Antennas Propag., vol. 38, no. 6, pp. 794799, Jun 1990.

[13] O. Ozgun, G. Apaydin, M. Kuzuoglu, L. Sevgi, "PETOOL: MATLABbased one-way and two-way split-step parabolic equation tool for radiowave propagation over variable terrain", Computer Physics Coтmunications, vol. 182, issue 12, pp. 2638-2654, Dec 2011.

[14] S. S. Mentes, Z. Kaymaz, "Investigation of Surface Duct Conditions over Istanbul, Turkey," J. Appl. Meteor., vol. 46, pp 318-338, 2007.

[15] "ITU UKSG3 CP(09): Information Document on The Modelling of Meteorological Parameters Using Radiosonde Data," Jan. 2009.

[16] "ITU-R's Rec. P. 837-6: Characteristics of precipitation for propagation modeling," ITU, Feb. 2012.

[17] "ITU-R's Rec. P. 838-3: Specific attenuation model for rain for use in prediction methods," ITU, 2005 